Published in: Potential Analysis 20 (2004), 285-302.

\title{
Non-differentiable Skew Convolution Semigroups and Related Ornstein-Uhlenbeck Processes
}

\author{
DONALD A. DAWSON ${ }^{1}$ \\ School of Mathematics and Statistics, Carleton University, 1125 Colonel By Drive, \\ Ottawa, Canada K1S 5B6 (e-mail: ddawson@math.carleton.ca) \\ ZENGHU LI ${ }^{2}$ \\ Department of Mathematics, Beijing Normal University, Beijing 100875, P.R. China \\ (e-mail: lizh@email.bnu.edu.cn)
}

\begin{abstract}
It is proved that a general non-differentiable skew convolution semigroup associated with a strongly continuous semigroup of linear operators on a real separable Hilbert space can be extended to a differentiable one on the entrance space of the linear semigroup. A càdlàg strong Markov process on an enlargement of the entrance space is constructed from which we obtain a realization of the corresponding Ornstein-Uhlenbeck process. Some explicit characterizations of the entrance spaces for special linear semigroups are given.
\end{abstract}

Mathematics Subject Classifications (2000): Primary 60J35; Secondary 60H15

Key words and phrases: skew convolution semigroup, differentiable extension, generalized Ornstein-Uhlenbeck process, right continuous realization.

\section{Introduction}

Suppose that $(S,+)$ is a Hausdorff topological semigroup and $\left(Q_{t}\right)_{t \geq 0}$ is a transition semigroup on $S$ satisfying

$$
Q_{t}\left(x_{1}+x_{2}, \cdot\right)=Q_{t}\left(x_{1}, \cdot\right) * Q_{t}\left(x_{2}, \cdot\right), \quad t \geq 0, x_{1}, x_{2} \in S
$$

where "*" denotes the convolution operation. A family of probability measures $\left(\mu_{t}\right)_{t \geq 0}$ on $S$ is called a skew convolution semigroup (SC-semigroup) associated with $\left(Q_{t}\right)_{t \geq 0}$ if it satisfies

$$
\mu_{r+t}=\left(\mu_{r} Q_{t}\right) * \mu_{t}, \quad r, t \geq 0
$$

This equation is of interest since it is satisfied if and only if

$$
Q_{t}^{\mu}(x, \cdot):=Q_{t}(x, \cdot) * \mu_{t}(\cdot), \quad t \geq 0, x \in S,
$$

\footnotetext{
${ }^{1}$ Supported by an NSERC Research Grant and a Max Planck Award.

${ }^{2}$ Supported by the NSFC (No. 10131040 and No. 10121101).
} 
defines another transition semigroup $\left(Q_{t}^{\mu}\right)_{t \geq 0}$ on $S$. (Note that $(1.1)$ implies $(\mu * \nu) Q_{t}=\left(\mu Q_{t}\right) *$ $\left(\nu Q_{t}\right)$ for probability measures $\mu$ and $\nu$ on $S$.) This fact was first observed in $[5,6]$ when $S=M(E)$ is the space of all finite Borel measures on a metrizable space $E$; see also [8, Theorem 2.1]. In that case, $\left(Q_{t}\right)_{t \geq 0}$ corresponds to a measure-valued branching process and $\left(Q_{t}^{\mu}\right)_{t \geq 0}$ corresponds to an immigration process.

In this work, we shall consider the formulation in another special situation, where $S=H$ is a real separable Hilbert space and $Q_{t}(x, \cdot) \equiv \delta_{T_{t} x}$ for a strongly continuous semigroup of bounded linear operators $\left(T_{t}\right)_{t \geq 0}$ on $H$. In this case, we can rewrite (1.2) as

$$
\mu_{r+t}=\left(T_{t} \mu_{r}\right) * \mu_{t}, \quad r, t \geq 0,
$$

and the transition semigroup $\left(Q_{t}^{\mu}\right)_{t \geq 0}$ is given by

$$
Q_{t}^{\mu} f(x):=\int_{H} f\left(T_{t} x+y\right) \mu_{t}(d y), \quad x \in H, f \in B(H),
$$

where $B(H)$ denotes the totality of bounded Borel measurable functions on $H$. The semigroup $\left(Q_{t}^{\mu}\right)_{t \geq 0}$ defined by (1.5) is called a generalized Mehler semigroup associated with $\left(T_{t}\right)_{t \geq 0}$, which corresponds to a generalized Ornstein-Uhlenbeck process (OU-process). This definition of the generalized Mehler semigroup was given by Bogachev et al [1]. They also gave a characterization for the SC-semigroup $\left(\mu_{t}\right)_{t \geq 0}$ under the assumption that the function $t \mapsto \hat{\mu}_{t}(a)$ is differentiable at $t=0$, where $\hat{\mu}_{t}(a)$ denotes the characteristic functional of $\mu_{t}$. It is known that for a general SC-semigroup $\left(\mu_{t}\right)_{t \geq 0}$ defined by (1.4) the function $t \mapsto \hat{\mu}_{t}(a)$ is not necessarily differentiable at $t=0$; see e.g. [?, 11, 12]. A simple and nice necessary and sufficient condition for an SCsemigroup to be differentiable was given in [11] in the setting of cylindrical probability measures. In [1] it was shown that a differentiable cylindrical Gaussian SC-semigroup can be extended into a real Gaussian SC-semigroup in an enlargement of $H$ and the corresponding OU-process was constructed as the strong solution to a stochastic differential equation. Those results were extended to the general non-Gaussian case in [4]. A characterization for general SC-semigroups $\left(\mu_{t}\right)_{t \geq 0}$ was given in [?], where it was also observed that the OU-processes corresponding to a non-differentiable SC-semigroup usually have no right continuous realizations. This property is similar to that of the immigration processes studied in $[6,7,8]$ and represents a departure from the theory of well-studied classes of OU-processes in $[1,4]$.

The main purpose of this paper is to study the construction of OU-processes corresponding to non-differentiable SC-semigroups. We shall see that, under a moment assumption, a general SCsemigroup can be decomposed as the convolution of a centered SC-semigroup and a degenerate one. For this reason, we shall only consider centered SC-semigroups. In section 2, we derive from the results of Dawson et al [?] that each centered SC-semigroup is uniquely determined by an infinitely divisible probability measure on the entrance space $\tilde{H}$ for the semigroup $\left(T_{t}\right)_{t \geq 0}$, which is an enlargement of $H$. In section 3 , it is shown that a general non-differentiable centered SCsemigroup can always be extended to a differentiable one on the entrance space $\tilde{H}$. In section 4, we use a modification of the argument of Fuhrman and Röckner [4] to construct a càdlàg and strong Markov OU-process $\left\{\bar{X}_{t}: t \geq 0\right\}$ on a further extension $\bar{H}$ of $\tilde{H}$. We also show that, if $\bar{X}_{0} \in H$, then $\bar{X}_{t} \in H$ almost surely for every $t \geq 0$ and $\left\{1_{H}\left(\bar{X}_{t}\right) \bar{X}_{t}: t \geq 0\right\}$ is an OU-process with transition semigroup $\left(Q_{t}^{\mu}\right)_{t \geq 0}$ defined by (1.5). Those results provide an approach to the study of non-differentiable generalized Mehler semigroups with which one can reduce some of their analysis to the existing framework of [1] and [4]. However, this approach 
should not convince the reader that non-differentiable generalized Mehler semigroups do not bear particular consideration on their own. In fact, there are some cases where the natural state space of the OU-process is $H$ and the introduction of $\tilde{H}$ and $\bar{H}$ seems unnatural and artificial. For example, an OU-process on $L^{2}(0, \infty)$ with non-differentiable SC-semigroup represents the fluctuation density of a catalytic branching processes with immigration; see [?]. In this case, it is rather unnatural to take $\bar{L}^{2}(0, \infty)$ as the state space. We provide some explicit characterization for the non-negative elements of $\tilde{L}^{2}\left(\mathbb{R}^{d}\right)$ and $\tilde{L}^{2}(0, \infty)$ in section 5 . The explicit characterization for all elements of $\bar{L}^{2}\left(\mathbb{R}^{d}\right)$ and $\bar{L}^{2}(0, \infty)$ seems much more sophisticated.

\section{Non-differentiable semigroups}

Suppose that $H$ is a real separable Hilbert space with dual space $H^{*}$ and $\left(T_{t}\right)_{t \geq 0}$ is a strongly continuous semigroup of linear operators on $H$ with dual $\left(T_{t}^{*}\right)_{t \geq 0}$. Let $\left(\mu_{t}\right)_{t \geq 0}$ be an SC-semigroup defined by (1.4) satisfying the moment condition

$$
\int_{H^{\circ}}\|x\|^{2} \mu_{t}(d x)<\infty, \quad t \geq 0
$$

where $H^{\circ}=H \backslash\{0\}$. Then we may define an $H$-valued path $\left(b_{t}\right)_{t \geq 0}$ by Bochner integrals

$$
b_{t}:=\int_{H^{\circ}} x \mu_{t}(d x), \quad t \geq 0,
$$

and define $\mu_{t}^{c}=\delta_{-b_{t}} * \mu_{t}$. It is easy to check that both $\left(\delta_{b_{t}}\right)_{t \geq 0}$ and $\left(\mu_{t}^{c}\right)_{t \geq 0}$ are SC-semigroups associated with $\left(T_{t}\right)_{t \geq 0}$ and $\mu_{t}=\mu_{t}^{c} * \delta_{b_{t}}$. That is, under the moment assumption, a general SCsemigroup can be decomposed as the convolution of a centered SC-semigroup and a degenerate one. For this reason, we shall only discuss centered SC-semigroups in the sequel.

Since $\left(T_{t}\right)_{t \geq 0}$ is strongly continuous, there are constants $c_{0} \geq 0$ and $b_{0} \geq 0$ such that $\left\|T_{t}\right\| \leq c_{0} e^{b_{0} t}$. Let $\left(U_{\alpha}\right)_{\alpha>b_{0}}$ denote the resolvent of $\left(T_{t}\right)_{t \geq 0}$ and let $A$ denote its generator with domain $D(A)=U_{\alpha} H \subset H$. An $H$-valued path $\tilde{x}=\{\tilde{x}(s): s>0\}$ is called an entrance path for the semigroup $\left(T_{t}\right)_{t \geq 0}$ if it satisfies $\tilde{x}(r+t)=T_{t} \tilde{x}(r)$ for all $r, t>0$. Let $E$ denote the set of all entrance paths for $\left(T_{t}\right)_{t \geq 0}$. We say $\tilde{x} \in E$ is closable if there is an element $\tilde{x}(0) \in H$ such that $\tilde{x}(s)=T_{s} \tilde{x}(0)$ for all $s>0$; and we say it is locally square integrable if

$$
\int_{0}^{l}\|\tilde{x}(s)\|^{2} d s<\infty
$$

for some $l>0$.

Lemma 2.1 For any $\tilde{x} \in E$, (2.2) holds for some $l>0$ if and only if it holds for all $l>0$; and if and only if

$$
\int_{0}^{\infty} e^{-2 b s}\|\tilde{x}(s)\|^{2} d s<\infty
$$

for all $b>b_{0}$. 
Proof. Suppose that (2.2) holds for some $l_{0}>0$. Let $l>0$ and let $n \geq 1$ be an integer such that $n l_{0} \geq l$. Then

$$
\begin{aligned}
\int_{0}^{l}\|\tilde{x}(s)\|^{2} d s & \leq \int_{0}^{n l_{0}}\|\tilde{x}(s)\|^{2} d s \\
& =\sum_{k=0}^{n-1} \int_{0}^{l_{0}}\left\|T_{k l_{0}} \tilde{x}(s)\right\|^{2} d s \\
& \leq \sum_{k=0}^{n-1} c_{0}^{2} e^{2 k b_{0} l_{0}} \int_{0}^{l_{0}}\|\tilde{x}(s)\|^{2} d s \\
& <\infty .
\end{aligned}
$$

Thus (2.2) holds for all $l>0$. On the other hand, for any $b>b_{0}$,

$$
\begin{aligned}
\int_{0}^{\infty} e^{-2 b s}\|\tilde{x}(s)\|^{2} d s & =\sum_{k=0}^{\infty} e^{-2 k b l_{0}} \int_{0}^{l_{0}} e^{-2 b s}\left\|T_{k l_{0}} \tilde{x}(s)\right\|^{2} d s \\
& \leq \sum_{k=0}^{\infty} c_{0}^{2} e^{-2 k\left(b-b_{0}\right) l_{0}} \int_{0}^{l_{0}} e^{-2 b s}\|\tilde{x}(s)\|^{2} d s \\
& <\infty .
\end{aligned}
$$

That is, (2.3) holds for all $b>b_{0}$. The remaining assertions are obvious.

Let $\tilde{H}$ denote the set of all locally square integrable entrance paths for $\left(T_{t}\right)_{t \geq 0}$. We shall call $\tilde{H}$ the entrance space for $\left(T_{t}\right)_{t \geq 0}$. For any fixed $b>b_{0}$, we may define an inner product on $\tilde{H}$ by

$$
\langle\tilde{x}, \tilde{y}\rangle_{\sim}:=\int_{0}^{\infty} e^{-2 b s}\langle\tilde{x}(s), \tilde{y}(s)\rangle d s, \quad \tilde{x}, \tilde{y} \in \tilde{H} .
$$

Let $\|\cdot\|_{\sim}$ denote the norm induced by this inner product. The proof of the following result was suggested to us by W. Sun.

Lemma 2.2 The normed space $\left(\tilde{H},\|\cdot\|_{\sim}\right)$ is complete, so $\left(\tilde{H},\langle\cdot, \cdot\rangle_{\sim}\right)$ is a Hilbert space.

Proof. Suppose $\left\{\tilde{x}_{n}\right\} \subset \tilde{H}$ is a Cauchy sequence under the norm $\|\cdot\|_{\sim}$, that is,

$$
\left\|\tilde{x}_{n}-\tilde{x}_{m}\right\|_{\sim}=\int_{0}^{\infty} e^{-2 b s}\left\|\tilde{x}_{n}(s)-\tilde{x}_{m}(s)\right\|^{2} d s \rightarrow 0
$$

as $m, n \rightarrow \infty$. For each $t>0$,

$$
\begin{aligned}
\left\|\tilde{x}_{n}(t)-\tilde{x}_{m}(t)\right\|^{2} & =t^{-1} \int_{0}^{t}\left\|\tilde{x}_{n}(t)-\tilde{x}_{m}(t)\right\|^{2} d s \\
& =t^{-1} \int_{0}^{t}\left\|T_{t-s}\left(\tilde{x}_{n}(s)-\tilde{x}_{m}(s)\right)\right\|^{2} d s \\
& \leq c_{0}^{2} t^{-1} e^{2 b t} \int_{0}^{t} e^{-2 b s}\left\|\tilde{x}_{n}(s)-\tilde{x}_{m}(s)\right\|^{2} d s .
\end{aligned}
$$

Then the limit $\tilde{x}(t)=\lim _{n \rightarrow \infty} \tilde{x}_{n}(t)$ exists in $H$. Since $T_{s}$ is a continuous operator on $H$, for $s>0$,

$$
T_{s} \tilde{x}(t)=\lim _{n \rightarrow \infty} T_{s} \tilde{x}_{n}(t)=\lim _{n \rightarrow \infty} \tilde{x}_{n}(t+s)=\tilde{x}(t+s)
$$


that is, $\tilde{x}=\{\tilde{x}(t): t>0\}$ is an entrance path for $\left(T_{t}\right)_{t \geq 0}$. For $\varepsilon>0$, choose large enough $N \geq 1$ such that

$$
\left\|\tilde{x}_{n}-\tilde{x}_{m}\right\|_{\sim}^{2}=\int_{0}^{\infty} e^{-2 b s}\left\|\tilde{x}_{n}(s)-\tilde{x}_{m}(s)\right\|^{2} d s<\varepsilon
$$

for $m, n \geq N$. By Fatou's lemma we get

$$
\int_{0}^{\infty} e^{-2 b s}\left\|\tilde{x}_{n}(s)-\tilde{x}(s)\right\|^{2} d s \leq \liminf _{m \rightarrow \infty} \int_{0}^{\infty} e^{-2 b s}\left\|\tilde{x}_{n}(s)-\tilde{x}_{m}(s)\right\|^{2} d s \leq \varepsilon .
$$

It follows that

$$
\int_{0}^{\infty} e^{-2 b s}\|\tilde{x}(s)\|^{2} d s \leq \int_{0}^{\infty} e^{-2 b s}\left\|\tilde{x}_{n}(s)\right\|^{2} d s+\int_{0}^{\infty} e^{-2 b s}\left\|\tilde{x}_{n}(s)-\tilde{x}(s)\right\|^{2} d s<\infty .
$$

Then $\tilde{x} \in \tilde{H}$ and $\lim _{n \rightarrow \infty}\left\|x_{n}-x\right\|_{\sim}^{2}=0$.

Lemma 2.3 The map $J: x \mapsto\left\{T_{s} x: s>0\right\}$ from $(H,\|\cdot\|)$ to $\left(\tilde{H},\|\cdot\|_{\sim}\right)$ is a continuous dense embedding and hence $\left(\tilde{H},\|\cdot\|_{\sim}\right)$ is separable.

Proof. Since $x=\lim _{t \rightarrow 0^{+}} T_{t} x$, the map $J: x \mapsto\left\{T_{s} x: s>0\right\}$ is injective. If $\lim _{n \rightarrow \infty} x_{n}=$ $x \in H$, then

$$
\int_{0}^{\infty} e^{-2 b s}\left\|T_{s} x_{n}-T_{s} x\right\|^{2} d s \leq c_{0}^{2}\left\|x_{n}-x\right\|^{2} \cdot \int_{0}^{\infty} e^{-2\left(b-b_{0}\right) s} d s \rightarrow 0
$$

as $n \rightarrow \infty$. Thus $J$ is a continuous embedding. For an arbitrary $\tilde{x} \in \tilde{H}$ we have

$$
\begin{aligned}
\|J \tilde{x}(t)-\tilde{x}\|_{\sim}^{2}= & \int_{0}^{\infty} e^{-2 b s}\left\|T_{t} \tilde{x}(s)-\tilde{x}(s)\right\|^{2} d s \\
= & \int_{0}^{r} e^{-2 b s}\left\|T_{t} \tilde{x}(s)-\tilde{x}(s)\right\|^{2} d s+\int_{r}^{\infty} e^{-2 b s}\left\|T_{s-r}\left[T_{t} \tilde{x}(r)-\tilde{x}(r)\right]\right\|^{2} d s \\
\leq & 2\left(c_{0}^{2} e^{2 b_{0} t}+1\right) \int_{0}^{r} e^{-2 b s}\|\tilde{x}(s)\|^{2} d s \\
& +c_{0}^{2} e^{-2 b_{0} r}\left\|T_{t} \tilde{x}(r)-\tilde{x}(r)\right\|^{2} \int_{r}^{\infty} e^{-2\left(b-b_{0}\right) s} d s .
\end{aligned}
$$

Observe that the first integral on the right hand side goes to zero as $r \rightarrow 0^{+}$and for fixed $r>0$ the second term goes to zero as $t \rightarrow 0^{+}$. Then we have $\|J \tilde{x}(t)-\tilde{x}\|_{\sim} \rightarrow 0$ as $t \rightarrow 0$, and $J H$ is dense in $\tilde{H}$. Since $H$ is separable, so is $\tilde{H}$.

Theorem 2.1 A family $\left(\mu_{t}\right)_{t \geq 0}$ of centered probability measures on $H$ satisfying (2.1) is an $S C$-semigroup associated with $\left(T_{t}\right)_{t \geq 0}$ if and only if its characteristic functionals are given by

$$
\hat{\mu}_{t}(a)=\exp \left\{\int_{0}^{t} \log \hat{\nu}_{s}(a) d s\right\}, \quad t \geq 0, a \in H^{*},
$$

where $\left(\nu_{s}\right)_{s>0}$ is a family of centered infinitely divisible probability measures on $H$ satisfying $\nu_{r+t}=T_{t} \nu_{r}$ for all $r, t>0$ and

$$
\int_{0}^{t} d s \int_{H^{\circ}}\|x\|^{2} \nu_{s}(d x)<\infty, \quad t \geq 0
$$

and $\log \hat{\nu}_{s}(\cdot)$ denotes the unique continuous function on $H^{*}$ with $\log \hat{\nu}_{s}(0)=0$ and $\hat{\nu}_{s}(a)=$ $\exp \left\{\log \hat{\nu}_{s}(a)\right\}$ for all $a \in H^{*}$. 
Proof. It is well-known that the second moment of a centered infinitely divisible probability measure only involves the Gaussian covariance operator and the Lévy measure. If the centered probability measures $\left(\mu_{t}\right)_{t \geq 0}$ and $\left(\nu_{s}\right)_{s>0}$ are related by $(2.5)$, the Gaussian covariance operators and Lévy measures of $\left(\mu_{t}\right)_{t \geq 0}$ can be represented as integrals of those of $\left(\nu_{s}\right)_{s>0}$. This observation yields that

$$
\int_{H^{\circ}}\langle x, a\rangle^{2} \mu_{t}(d x)=\int_{0}^{t} d s \int_{H^{\circ}}\langle x, a\rangle^{2} \nu_{s}(d x), \quad t \geq 0, a \in H^{*} .
$$

Let $\left\{e_{n}: n=1,2, \ldots\right\}$ be an orthonormal basis of $H=H^{*}$. Applying the above equation to each $e_{n}$ and taking the summation we see

$$
\int_{H^{\circ}}\|x\|^{2} \mu_{t}(d x)=\int_{0}^{t} d s \int_{H^{\circ}}\|x\|^{2} \nu_{s}(d x), \quad t \geq 0 .
$$

Thus conditions (2.1) and (2.6) are equivalent for the probability measures $\left(\mu_{t}\right)_{t \geq 0}$ and $\left(\nu_{s}\right)_{s>0}$ related by (2.5). Suppose $\left(\mu_{t}\right)_{t \geq 0}$ is given by $(2.5)$ with the centered infinitely divisible probabilities $\left(\nu_{s}\right)_{s>0}$ satisfying $\nu_{r+t}=T_{t} \nu_{r}$ for all $r, t>0$. Then $\left(\mu_{t}\right)_{t \geq 0}$ is a centered SC-semigroup by [?, Theorem 2.3]. Conversely, by [?, Theorems 2.1 and 2.2] any SC-semigroup $\left(\mu_{t}\right)_{t \geq 0}$ has the expression (2.5) up to the convolution of a family of degenerate probability measures $\left(\delta_{b_{t}}\right)_{t \geq 0}$. If $\left(\mu_{t}\right)_{t \geq 0}$ is a centered SC-semigroup, we must have $b_{t}=0$ for all $t \geq 0$.

Theorem 2.2 A family $\left(\mu_{t}\right)_{t \geq 0}$ of centered probability measures on $H$ satisfying (2.1) is an $S C$-semigroup associated with $\left(T_{t}\right)_{t \geq 0}$ if and only if its characteristic functionals are given by

$$
\hat{\mu}_{t}(a)=\exp \left\{-\int_{0}^{t} \psi_{s}(a) d s\right\}, \quad t \geq 0, a \in H^{*}
$$

where $\psi_{s}(\cdot)$ denotes the unique continuous function on $H^{*}$ with $\psi_{s}(0)=0$ and

$$
\exp \left\{-\psi_{s}(a)\right\}=\int_{\tilde{H}} e^{i\langle\tilde{x}(s), a\rangle} \lambda_{0}(d \tilde{x}), \quad s>0, a \in H^{*},
$$

where $\lambda_{0}$ is a centered infinitely divisible probability measure on $\tilde{H}$ satisfying

$$
\int_{\tilde{H}}\|\tilde{x}\|_{\sim}^{2} \lambda_{0}(d \tilde{x})<\infty .
$$

Proof. Let $\left(\nu_{s}\right)_{s>0}$ be given as in Theorem 2.1. In the terminology of Markov processes, $\left(\nu_{s}\right)_{s>0}$ is a probability entrance law for the Markov process $\left\{T_{t} x: t \geq 0\right\}$ with deterministic motion. Let $E_{0}=H^{(0, \infty)}$ be the totality of paths $\{w(t): t>0\}$ from $(0, \infty)$ to $H$. We endow $E_{0}$ with the $\sigma$-algebra $\mathcal{E}_{0}$ generated by the maps $w \mapsto w(s), s>0$. By Kolmogorov's existence theorem, there is a unique probability measure $\lambda_{0}$ on $E_{0}$ so that $\{w(t): t>0\}$ under $\lambda_{0}$ is a Markov process with the same transition semigroup as the process $\left\{T_{t} x: t \geq 0\right\}$ and $\nu_{s}$ is the image of $\lambda_{0}$ under $w \mapsto w(s)$; see e.g. Sharpe [13, p.6]. Because of the special deterministic motion mechanism of $\left\{T_{t} x: t \geq 0\right\}$ we may assume that $\lambda_{0}$ is supported by the entrance paths $E$. Let $\mathcal{E}_{0}(E)$ and $\mathcal{E}_{0}(\tilde{H})$ denote respectively the traces of $\mathcal{E}_{0}$ on $E$ and $\tilde{H}$. Since $w \mapsto\|w(s)\|^{2}$ is clearly a non-negative $\mathcal{E}_{0}(E)$-measurable function on $E$,

$$
w \mapsto\|w\|_{\sim}:=\int_{0}^{\infty} e^{-2 b s}\|w(s)\|^{2} d s
$$


is an $\mathcal{E}_{0}(E)$-measurable function on $E$ taking values in $[0, \infty]$. It is also easy to check that $\mathcal{E}_{0}(\tilde{H})$ coincides with the Borel $\sigma$-algebra $\mathcal{B}(\tilde{H})$ induced by the norm $\|\cdot\|_{\sim}$. Since $\left(\nu_{s}\right)_{s>0}$ satisfies $(2.6)$, we have

$$
\begin{aligned}
\int_{E}\|w\|_{\sim}^{2} \lambda_{0}(d w) & =\int_{E} \lambda_{0}(d w) \int_{0}^{\infty} e^{-2 b s}\|w(s)\|^{2} d s \\
& =\int_{0}^{\infty} d s \int_{H} e^{-2 b s}\|x\|^{2} \nu_{s}(d x) \\
& =\sum_{n=0}^{\infty} \int_{0}^{1} d s \int_{H} e^{-2 b(n+s)}\left\|T_{n} x\right\|^{2} \nu_{s}(d x) \\
& \leq c_{0}^{2} \sum_{n=0}^{\infty} e^{-2\left(b-b_{0}\right) n} \int_{0}^{1} d s \int_{H} e^{-2 b s}\|x\|^{2} \nu_{s}(d x) \\
& <\infty
\end{aligned}
$$

so $\lambda_{0}$ is supported by $\tilde{H}$ and (2.10) holds. The infinite divisibility of $\lambda_{0}$ follows immediately from that of $\nu_{s}$.

\section{Differentiable extensions}

For a general SC-semigroup given by Theorem 2.2, the function $t \mapsto \hat{\mu}_{t}(a)$ is not necessarily differentiable at $t=0$. However, if $\nu_{0}$ is a centered infinitely divisible probability measure on $H$ satisfying

$$
\int_{H^{\circ}}\|x\|^{2} \nu_{0}(d x)<\infty
$$

then

$$
\hat{\mu}_{t}(a)=\exp \left\{\int_{0}^{t} \log \hat{\nu}_{0}\left(T_{s}^{*} a\right) d s\right\}, \quad t \geq 0, a \in H^{*},
$$

defines a centered SC-semigroup $\left(\mu_{t}\right)_{t \geq 0}$ such that $t \mapsto \hat{\mu}_{t}(a)$ is differentiable at $t=0$ for all $a \in H^{*}$. In the sequel, we shall call $\left(\mu_{t}\right)_{t \geq 0}$ a differentiable SC-semigroup if it is given by (3.2). We shall discuss how to extend a general SC-semigroup on $H$ to a differentiable one on the entrance space $\tilde{H}$. For any strongly continuous linear semigroup $\left(T_{t}\right)_{t \geq 0}$ on $H$,

$$
\left(\tilde{T}_{t} \tilde{x}\right)(s)=\tilde{x}(t+s), \quad s, t>0
$$

defines a semigroup of linear operators $\left(\tilde{T}_{t}\right)_{t \geq 0}$ on $\tilde{H}$. Since

$$
\left\|\tilde{T} \tilde{x}_{t}^{2}\right\|_{\sim}^{\infty}=\int_{0}^{-2 b s}\|\tilde{x}(t+s)\|^{2} d s \leq\left\|T_{t}\right\|^{2} \int_{0}^{\infty} e^{-2 b s}\|\tilde{x}(s)\|^{2} d s
$$

we have $\left\|\tilde{T}_{t}\right\|_{\sim} \leq\left\|T_{t}\right\|$. Let $\left(\tilde{U}_{\alpha}\right)_{\alpha>b_{0}}$ denote the resolvent of $\left(\tilde{T}_{t}\right)_{t \geq 0}$ and let $\tilde{A}$ denote its generator with domain $D(\tilde{A})=\tilde{U}_{\alpha} \tilde{H} \subset \tilde{H}$.

Lemma 3.1 Let $J$ be defined as in Lemma 2.3. Then $J T_{t} x=\tilde{T}_{t} J x$ for all $t \geq 0$ and $x \in H$ and $\left(\tilde{T}_{t}\right)_{t \geq 0}$ is a strongly continuous semigroup of linear operators on $\tilde{H}$. 
Proof. For $t \geq 0$ and $x \in H$ we have

$$
J T_{t} x=\left\{T_{s} T_{t} x: s>0\right\}=\left\{T_{t} T_{s} x: s>0\right\}=\tilde{T}_{t} J x,
$$

giving the first assertion. By the proof of Lemma 2.3, $\left\|\tilde{T}_{t} \tilde{x}-\tilde{x}\right\|_{\sim}=\|J \tilde{x}(t)-\tilde{x}\|_{\sim} \rightarrow 0$ as $t \rightarrow 0$, that is, $\left(\tilde{T}_{t}\right)_{t \geq 0}$ is strongly continuous.

Lemma 3.2 We have $\tilde{U}_{\alpha} \tilde{x}=\left\{U_{\alpha} \tilde{x}(s): s>0\right\}$ and $\tilde{A} \tilde{U}_{\alpha} \tilde{x}=\left\{A U_{\alpha} x(s): s>0\right\}$ for all $\tilde{x} \in \tilde{H}$.

Proof. The first assertion follows as we observe that

$$
\tilde{U}_{\alpha} \tilde{x}(s)=\int_{0}^{\infty} e^{-\alpha s} \tilde{T}_{t} \tilde{x}(s) d t=\int_{0}^{\infty} e^{-\alpha s} T_{t} \tilde{x}(s) d t=U_{\alpha} \tilde{x}(s)
$$

and the second follows from the equality $\tilde{A} \tilde{U}_{\alpha} \tilde{x}=\alpha \tilde{U}_{\alpha} \tilde{x}-\tilde{x}$.

Theorem 3.1 All centered SC-semigroups associated with $\left(T_{t}\right)_{t \geq 0}$ satisfying (2.1) are differentiable if and only if all its locally square integrable entrance paths are closable.

Proof. Suppose that all entrance paths $\tilde{x} \in \tilde{H}$ are closable and $\left(\mu_{t}\right)_{t \geq 0}$ is an SC-semigroup given by (2.8). To each $\tilde{x} \in \tilde{H}$ there corresponds some $\tilde{x}(0) \in H$ such that $\tilde{x}(s)=T_{s} \tilde{x}(0)$ for all $s>0$. This element is apparently determined by $\tilde{x}$ uniquely. Letting $\nu_{0}$ be the image of $\lambda_{0}$ under the map $\tilde{x} \mapsto \tilde{x}(0)$ we get (3.2). Conversely, if $\tilde{x}=\{\tilde{x}(s): s>0\} \in \tilde{H}$ is not closable, then

$$
\hat{\mu}_{t}(a)=\exp \left\{-\frac{1}{2} \int_{0}^{t}\langle\tilde{x}(s), a\rangle^{2} d s\right\}, \quad t \geq 0, a \in H^{*},
$$

defines a non-differentiable SC-semigroup.

Theorem 3.2 All entrance paths for $\left(\tilde{T}_{t}\right)_{t \geq 0}$ are closable.

Proof. Suppose that $\bar{x}=\{\bar{x}(u): u>0\}$ is an entrance path for $\left(\tilde{T}_{t}\right)_{t \geq 0}$, where each $\bar{x}(u)=\{\bar{x}(u, s): s>0\} \in \tilde{H}$ is an entrance path for $\left(T_{t}\right)_{t \geq 0}$. Then we get

$$
\{\bar{x}(u, r+s): s>0\}=\left(\tilde{T}_{r} \bar{x}\right)(u)=\bar{x}(r+u)=\{\bar{x}(r+u, s): s>0\},
$$

where the first equality follows from (3.3) and the second one holds since $\bar{x}$ is an entrance path for $\left(\tilde{T}_{t}\right)_{t \geq 0}$. Setting $\bar{x}(0)=\{\bar{x}(s / 2, s / 2): s>0\}$ we have

$$
\tilde{T}_{u} \bar{x}(0)(s)=\bar{x}(s / 2, u+s / 2)=\bar{x}(u, s),
$$

where the first equality follows from (3.3) and the second one holds by (3.4). Thus $\tilde{T}_{u} \bar{x}(0)=\bar{x}(u)$, that is, $\bar{x}=\{\bar{x}(u): u>0\}$ is closed by $\bar{x}(0)$.

For the infinitely divisible probability measure $\lambda_{0}$ on $\tilde{H}$ given by Theorem 2.2 we have

$$
\hat{\lambda}_{0}(\tilde{a})=e^{-\tilde{\psi}_{0}(\tilde{a})}, \quad \tilde{a} \in \tilde{H}^{*},
$$

for a functional $\tilde{\psi}_{0}$ on $\tilde{H}^{*}$ with representation

$$
\tilde{\psi}_{0}(\tilde{a})=\frac{1}{2}\langle\tilde{R} a, a\rangle_{\sim}-\int_{\tilde{H}^{\circ}}\left(e^{i\langle\tilde{x}, \tilde{a}\rangle_{\sim}}-1-i\langle\tilde{x}, \tilde{a}\rangle_{\sim}\right) \tilde{M}(d \tilde{x}), \quad \tilde{a} \in \tilde{H}^{*},
$$

where $R$ is a nuclear operator on $\tilde{H}$ and $\|\tilde{x}\|_{\sim}^{2} \tilde{M}(d \tilde{x})$ is a finite measure on $\tilde{H}^{\circ}:=\tilde{H} \backslash\{0\}$; see e.g. $[10]$. 
Theorem 3.3 Let $\left(\mu_{t}\right)_{t \geq 0}$ be a centered SC-semigroup given by (2.8) and let $\tilde{\mu}_{t}=J \mu_{t}$. Then $\left(\tilde{\mu}_{t}\right)_{t \geq 0}$ is a differentiable centered SC-semigroup associated with $\left(\tilde{T}_{t}\right)_{t \geq 0}$ and

$$
\int_{\tilde{H}} e^{i\langle\tilde{x}, \tilde{a}\rangle_{\sim}} \tilde{\mu}_{t}(d \tilde{x})=\exp \left\{-\int_{0}^{t} \tilde{\psi}_{0}\left(\tilde{T}_{s}^{*} \tilde{a}\right) d s\right\}, \quad t \geq 0, \tilde{a} \in \tilde{H}^{*} .
$$

Proof. By Lemma 2.3, $J: H \mapsto \tilde{H}$ is an embedding. Thus $\left(\tilde{\mu}_{t}\right)_{t \geq 0}$ is an SC-semigroup associated with $\left(\tilde{T}_{t}\right)_{t \geq 0}$. Since $\left(T_{t}\right)_{t \geq 0}$ is a strongly continuous semigroup, for any $\tilde{a}=\{\tilde{a}(s)$ : $s>0\} \in \tilde{H}$ we have by dominated convergence

$$
\begin{aligned}
& \int_{H} \exp \left\{i \int_{0}^{\infty} e^{-2 b s}\left\langle T_{s} x, \tilde{a}(s)\right\rangle d s\right\} \mu_{t}(d x) \\
= & \lim _{n \rightarrow \infty} \int_{H} \exp \left\{i \sum_{k=1}^{\infty} n^{-1} e^{-2 b k / n}\left\langle T_{k / n} x, \tilde{a}(k / n)\right\rangle\right\} \mu_{t}(d x) \\
= & \lim _{n \rightarrow \infty} \int_{H} \exp \left\{i\left\langle x, \sum_{k=1}^{\infty} n^{-1} e^{-2 b k / n} T_{k / n}^{*} \tilde{a}(k / n)\right\rangle\right\} \mu_{t}(d x) \\
= & \lim _{n \rightarrow \infty} \exp \left\{\int_{0}^{t}\left[\log \int_{H} \exp \left\{i\left\langle x, \sum_{k=1}^{\infty} n^{-1} e^{-2 b k / n} T_{k / n}^{*} \tilde{a}(k / n)\right\rangle\right\} \nu_{s}(d x)\right] d s\right\} \\
= & \lim _{n \rightarrow \infty} \exp \left\{\int_{0}^{t}\left[\log \int_{H} \exp \left\{i \sum_{k=1}^{\infty} n^{-1} e^{-2 b k / n}\left\langle T_{k / n} x, \tilde{a}(k / n)\right\rangle\right\} \nu_{s}(d x)\right] d s\right\} \\
= & \exp \left\{\int_{0}^{t}\left[\log \int_{H} \exp \left\{i \int_{0}^{\infty} e^{-2 b u}\left\langle T_{u} x, \tilde{a}(u)\right\rangle d u\right\} \nu_{s}(d x)\right] d s\right\} .
\end{aligned}
$$

It follows that

$$
\int_{\tilde{H}} e^{i\langle\tilde{x}, \tilde{a}\rangle_{\sim}} J \mu_{t}(d \tilde{x})=\exp \left\{\int_{0}^{t}\left[\log \int_{\tilde{H}} e^{i\langle\tilde{x}, \tilde{a}\rangle_{\sim}} J \nu_{s}(d \tilde{x})\right] d s\right\}, \quad t \geq 0, \tilde{a} \in \tilde{H} .
$$

Recall from the proof of Theorem 2.2 that $\nu_{s}$ is the image of $\lambda_{0}$ under $\tilde{x} \mapsto \tilde{x}(s)$. Then $\tilde{T}_{s} \lambda_{0}=J \nu_{s}$ and (3.8) follows from (3.9) and (3.6).

Theorem 3.4 Let $\left(\tilde{\mu}_{t}\right)_{t \geq 0}$ be a centered $S C$-semigroup associated with $\left(\tilde{T}_{t}\right)_{t \geq 0}$ satisfying

$$
\int_{\tilde{H}^{\circ}}\|\tilde{x}\|^{2} \tilde{\mu}_{t}(d \tilde{x})<\infty, \quad t \geq 0
$$

Then there is a centered SC-semigroup $\left(\mu_{t}\right)_{t \geq 0}$ associated with $\left(T_{t}\right)_{t \geq 0}$ satisfying (2.1) and $\tilde{\mu}_{t}=J \mu_{t}$ for each $t \geq 0$.

Proof. By Theorems 3.1 and 3.2, $\left(\tilde{\mu}_{t}\right)_{t \geq 0}$ is differentiable, so it has the expression (3.8) for an infinitely divisible probability $\lambda_{0}$ on $\tilde{H}$ defined by (3.6). Then we get $\left(\mu_{t}\right)_{t \geq 0}$ by Theorem 2.2 , which clearly satisfies the requirements.

By Theorems 3.3 and 3.4, centered SC-semigroups associated with $\left(T_{t}\right)_{t \geq 0}$ and those associated with $\left(\tilde{T}_{t}\right)_{t \geq 0}$ are in 1-1 correspondence. Therefore we may reduce some analysis of non-differentiable centered SC-semigroups to those of differentiable ones studied in $[1,4]$. 


\section{Ornstein-Uhlenbeck processes}

In this section, we discuss constructions of the OU-processes. By the results of the last section, a general centered SC-semigroup on $H$ can be extended to a differentiable one on the entrance space $\tilde{H}$. Then by Fuhrman and Röckner [4, Theorem 5.3], there is an extension $E$ of $\tilde{H}$ on which a càdlàg realization of the corresponding OU-process can be constructed. In the sequel, we shall give a modification of the arguments of Fuhrman and Röckner [4] which provides a smaller extension but still contains a càdlàg realization of the OU-process. Fix $\alpha>b_{0}$ and define an inner product on $\tilde{H}$ by

$$
\langle\tilde{x}, \tilde{y}\rangle_{-}=\int_{0}^{\infty} e^{-2 b s}\left\langle U_{\alpha} \tilde{x}(s), U_{\alpha} \tilde{y}(s)\right\rangle d s, \quad \tilde{x}, \tilde{y} \in \tilde{H} .
$$

Let $\|\cdot\|_{-}$be the corresponding norm and let $\bar{H}$ be the completion of $\tilde{H}$ with respect to $\|\cdot\|_{-}$.

Lemma 4.1 For $\tilde{x} \in \tilde{H}$ we have $\|\tilde{x}\|_{-} \leq\left\|U_{\alpha}\right\|\|\tilde{x}\|_{\sim}$, so the identity mapping $I$ from $\left(\tilde{H},\|\cdot\|_{\sim}\right)$ to $\left(\bar{H},\|\cdot\|_{-}\right)$is a continuous embedding.

Proof. By (2.4) and (4.1),

$$
\|\tilde{x}\|_{-}^{2}=\int_{0}^{\infty} e^{-2 b s}\left\|U_{\alpha} \tilde{x}(s)\right\|^{2} d s \leq\left\|U_{\alpha}\right\|^{2} \int_{0}^{\infty} e^{-2 b s}\|\tilde{x}(s)\|^{2} d s=\left\|U_{\alpha}\right\|^{2}\|\tilde{x}\|_{\sim}^{2},
$$

giving the desired estimate.

Note that the embedding of $\left(\tilde{H},\|\cdot\|_{\sim}\right)$ into $\left(\bar{H},\|\cdot\|_{-}\right)$is not necessarily Hilbert-Schmidt, so our extension is different from the one used in [4]. For $\tilde{x} \in \tilde{H}$ we have, by (4.1),

$$
\left\|\tilde{T}_{t} \tilde{x}\right\|_{-}^{2}=\int_{0}^{\infty} e^{-2 b s}\left\|U_{\alpha} T_{t} \tilde{x}(s)\right\|^{2} d s=\int_{0}^{\infty} e^{-2 b s}\left\|U_{\alpha} \tilde{x}(t+s)\right\|^{2} d s \leq e^{2 b t}\|\tilde{x}\|_{-}^{2} .
$$

Then each $\tilde{T}_{t}$ has a unique extension to a bounded linear operator $\bar{T}_{t}$ on $\bar{H}$. Since the semigroup property and strong continuity of $\left(\bar{T}_{t}\right)_{t \geq 0}$ hold on the dense subspace $\tilde{H}$ of $\bar{H}$, they also hold on $\bar{H}$, that is, the semigroup of linear operators $\left(\bar{T}_{t}\right)_{t \geq 0}$ extends $\left(\tilde{T}_{t}\right)_{t \geq 0}$. Let $\left(\bar{U}_{\alpha}\right)_{\alpha>b_{0}}$ denote the resolvent of $\left(\bar{T}_{t}\right)_{t \geq 0}$ and let $\bar{A}$ denote its generator with domain $D(\bar{A})=\bar{U}_{\alpha} \bar{H} \subset \bar{H}$. Then $D(\bar{A})$ is a Hilbert space with inner product norm $\|\cdot\|_{\bar{A}}$ defined by

$$
\|\bar{x}\|_{\bar{A}}=\|\bar{x}\|_{-}+\|\bar{A} \bar{x}\|_{-}, \quad \bar{x} \in D(\bar{A}) .
$$

Lemma 4.2 We have $\tilde{H} \subset D(\bar{A})$ and

$$
\|\bar{A} \tilde{y}\|_{-} \leq 2\left(\alpha^{2}\left\|U_{\alpha}\right\|^{2}+1\right)^{1 / 2}\|\tilde{y}\|_{\sim}, \quad \tilde{y} \in \tilde{H} .
$$

Consequently, the identity mapping I from $\left(\tilde{H},\|\cdot\|_{\sim}\right)$ to $\left(D(\bar{A}),\|\cdot\|_{\bar{A}}\right)$ is a continuous embedding.

Proof. Suppose that $\tilde{y} \in D(\tilde{A}) \subset D(\bar{A})$. Then $\tilde{y}=\tilde{U}_{\alpha} \tilde{x}$ for some $\tilde{x} \in \tilde{H}$. By (4.1) and Lemma 3.2,

$$
\begin{aligned}
\|\bar{A} \tilde{y}\|_{-}^{2} & =\left\|\tilde{A} \tilde{U}_{\alpha} \tilde{x}\right\|_{-}^{2} \\
& =\int_{0}^{\infty} e^{-2 b s}\left\|A U_{\alpha}^{2} \tilde{x}(s)\right\|^{2} d s \\
& =\int_{0}^{\infty} e^{-2 b s}\left\|\alpha U_{\alpha}^{2} \tilde{x}(s)-U_{\alpha} \tilde{x}(s)\right\|^{2} d s \\
& \leq 2\left(\alpha^{2}\left\|U_{\alpha}\right\|^{2}+1\right) \int_{0}^{\infty} e^{-2 b s}\left\|U_{\alpha} \tilde{x}(s)\right\|^{2} d s \\
& =2\left(\alpha^{2}\left\|U_{\alpha}\right\|^{2}+1\right)\|\tilde{y}\|_{\sim}^{2} .
\end{aligned}
$$


Since $D(\tilde{A})$ is a dense subset of $\left(\tilde{H},\|\cdot\|_{\sim}\right)$, we have $\tilde{H} \subset D(\bar{A})$ by (4.2) and the above inequality remains true for all $\tilde{y} \in \tilde{H}$.

Now suppose that $\left(\mu_{t}\right)_{t \geq 0}$ and $\left(\tilde{\mu}_{t}\right)_{t \geq 0}$ are the SC-semigroups described in Theorem 3.3. Let $\bar{\mu}_{t}$ be the unique probability measure on $\bar{H}$ whose restriction to $\tilde{H}$ is $\tilde{\mu}_{t}$. Then $\left(\bar{\mu}_{t}\right)_{t \geq 0}$ is an SC-semigroup associated with $\left(\bar{T}_{t}\right)_{t \geq 0}$. By $(3.8)$,

$$
\int_{\bar{H}} e^{i\langle\bar{x}, \bar{a}\rangle_{-}} \bar{\mu}_{t}(d \bar{x})=\exp \left\{-\int_{0}^{t} \tilde{\psi}_{0}\left(\bar{T}_{s}^{*} \bar{a}\right) d s\right\}, \quad t \geq 0, \bar{a} \in \bar{H}^{*} \subset \tilde{H}^{*},
$$

where $\tilde{\psi}_{0}(\cdot)$ is defined by (3.6). Let $\left(Q_{t}^{\bar{\mu}}\right)_{t \geq 0}$ be the generalized Mehler semigroup defined by (1.5) from $\left(\bar{T}_{t}\right)_{t \geq 0}$ and $\left(\bar{\mu}_{t}\right)_{t \geq 0}$. By [4, Theorem 5.1], there is a càdlàg $\tilde{H}$-valued process $\left\{\tilde{Y}_{t}: t \geq 0\right\}$ with $\tilde{Y}_{0}=0$ and with independent increments such that $\tilde{Y}_{t}-\tilde{Y}_{r}$ has distribution $\gamma_{t-r}$ with

$$
\hat{\gamma}_{t-r}(\tilde{a})=\exp \left\{-(t-r) \tilde{\psi}_{0}(\tilde{a})\right\}, \quad t \geq r \geq 0, \tilde{a} \in \tilde{H}^{*} .
$$

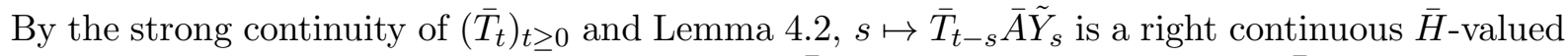
function of $s \in[0, t]$. Then for any given $\bar{x} \in \bar{H}$ we may define the càdlàg $\bar{H}$-valued process $\left\{\bar{X}_{t}: t \geq 0\right\}$ by

$$
\bar{X}_{t}=\bar{T}_{t} \bar{x}+\tilde{Y}_{t}+\int_{0}^{t} \bar{T}_{t-s} \bar{A} \tilde{Y}_{s} d s, \quad t \geq 0 .
$$

Lemma 4.3 The $\bar{H}$-valued random variable $\bar{X}_{t}$ has distribution $Q_{t}^{\bar{\mu}}(\bar{x}, \cdot)$ for every $t \geq 0$. In particular, if $\bar{x} \in J H$, then $\bar{X}_{t} \in J H$ a.s. for every $t \geq 0$.

Proof. We first prove that $\bar{X}_{t}^{(0)}:=\bar{X}_{t}-\bar{T}_{t} \bar{x}$ has distribution $\bar{\mu}_{t}(\cdot)=Q_{t}^{\bar{\mu}}(0, \cdot)$. By the right continuity of $s \mapsto \bar{T}_{t-s} \bar{A} \tilde{Y}_{s}$, we have

$$
\bar{X}_{t}^{(n)}:=\tilde{Y}_{t}+\frac{t}{n} \sum_{k=1}^{n} \bar{T}_{(1-k / n) t} \bar{A} \tilde{Y}_{k t / n} \rightarrow \tilde{Y}_{t}+\int_{0}^{t} \bar{T}_{t-s} \bar{A} \tilde{Y}_{s} d s=\bar{X}_{t}^{(0)}
$$

as $n \rightarrow \infty$. Let $D_{0}=0$ and $D_{k}=\bar{T}_{(1-n / n) t} \bar{A}+\cdots+\bar{T}_{(1-k / n) t} \bar{A}$. Then we have

$$
\begin{aligned}
\bar{X}_{t}^{(n)}= & \tilde{Y}_{t}+n^{-1} t\left[\left(D_{1}-D_{2}\right) \tilde{Y}_{t / n}+\cdots+\left(D_{n-1}-D_{n}\right) \tilde{Y}_{(n-1) t / n}+D_{n} \tilde{Y}_{n t / n}\right] \\
= & \left(\tilde{Y}_{n t / n}-\tilde{Y}_{(n-1) t / n}\right)+\cdots+\left(\tilde{Y}_{2 t / n}-\tilde{Y}_{t / n}\right)+\left(\tilde{Y}_{t / n}-\tilde{Y}_{0}\right) \\
& +n^{-1} t\left[D_{1}\left(\tilde{Y}_{t / n}-\tilde{Y}_{0}\right)+D_{2}\left(\tilde{Y}_{2 t / n}-\tilde{Y}_{t / n}\right)+\cdots\right. \\
& \left.+D_{n}\left(\tilde{Y}_{n t / n}-\tilde{Y}_{(n-1) t / n}\right)\right] \\
= & \left(I+n^{-1} t D_{1}\right)\left(\tilde{Y}_{t / n}-\tilde{Y}_{0}\right)+\left(I+n^{-1} t D_{2}\right)\left(\tilde{Y}_{2 t / n}-\tilde{Y}_{t / n}\right)+\cdots \\
& \left.+\left(I+n^{-1} t D_{n}\right)\left(\tilde{Y}_{n t / n}-\tilde{Y}_{(n-1) t / n}\right)\right] .
\end{aligned}
$$

It follows that

$$
\begin{aligned}
\boldsymbol{E} \exp \left\{i\left\langle\bar{X}_{t}^{(n)}, \bar{a}\right\rangle_{-}\right\} & =\boldsymbol{E} \exp \left\{i \sum_{k=1}^{n}\left\langle\left(I+n^{-1} t D_{k}\right)\left(\tilde{Y}_{k t / n}-\tilde{Y}_{(k-1) t / n}\right), \bar{a}\right\rangle_{-}\right\} \\
& =\boldsymbol{E} \exp \left\{i \sum_{k=1}^{n}\left\langle\left(\tilde{Y}_{k t / n}-\tilde{Y}_{(k-1) t / n}\right),\left(I+n^{-1} t D_{k}\right)^{*} \bar{a}\right\rangle_{-}\right\} \\
& =\exp \left\{-\frac{t}{n} \sum_{k=1}^{n} \tilde{\psi}_{0}\left(\left(I+n^{-1} t D_{k}\right)^{*} \bar{a}\right)\right\} .
\end{aligned}
$$


In view of $(3.7), \tilde{\psi}_{0}(\cdot)$ is uniformly continuous on any bounded subset of $\bar{H}^{*}$. Observe also that

$$
\begin{aligned}
& \left\|\bar{T}_{(1-k / n) t}^{*} \bar{a}-\left(I+n^{-1} t D_{k}\right)^{*} \bar{a}\right\|_{-} \\
= & \left\|\bar{T}_{(1-k / n) t}^{*} \bar{a}-\bar{a}-\frac{t}{n} \sum_{j=k}^{n} \bar{T}_{(1-j / n) t}^{*} \bar{A}^{*} \bar{a}\right\|_{\bar{H}} \\
\leq & \sum_{j=k}^{n} \int_{(1-j / n) t}^{(1-(j-1) / n) t}\left\|\bar{T}_{s}^{*} \bar{A}^{*} \bar{a}-\bar{T}_{(1-j / n) t}^{*} \bar{A}^{*} \bar{a}\right\|_{-} d s \\
\leq & t \cdot \sup \left\{\left\|\bar{T}_{t_{2}}^{*} \bar{A}^{*} \bar{a}-\bar{T}_{t_{1}}^{*} \bar{A}^{*} \bar{a}\right\|_{-}: 0 \leq t_{1}, t_{2} \leq t \text { and }\left|t_{2}-t_{1}\right|<t / n\right\},
\end{aligned}
$$

which goes to zero as $n \rightarrow \infty$. Thus we have

$$
\begin{aligned}
\boldsymbol{E} \exp \left\{i\left\langle\bar{X}_{t}^{(0)}, \bar{a}\right\rangle_{-}\right\} & =\lim _{n \rightarrow \infty} \boldsymbol{E} \exp \left\{i\left\langle\bar{X}_{t}^{(n)}, \bar{a}\right\rangle_{-}\right\} \\
& =\lim _{n \rightarrow \infty} \exp \left\{-\frac{t}{n} \sum_{k=1}^{n} \tilde{\psi}_{0}\left(\bar{T}_{(1-k / n) t}^{*} \bar{a}\right)\right\} \\
& =\exp \left\{-\int_{0}^{t} \tilde{\psi}_{0}\left(\bar{T}_{t-s}^{*} \bar{a}\right) d s\right\},
\end{aligned}
$$

so that $\bar{X}_{t}^{(0)}$ has distribution $Q_{t}^{\bar{\mu}}(0, \cdot)$. Therefore, $\bar{X}_{t}$ has distribution $Q_{t}^{\bar{\mu}}(\bar{x}, \cdot)$. If $\bar{x}=J x$ for some $x \in H$, then $\bar{T}_{t} \bar{x}=\tilde{T}_{t} J x=J T_{t} x \in J H$ by Lemma 3.1. Since $\bar{\mu}_{t}(\cdot)$ is supported by $J H$, so is $Q_{t}^{\bar{\mu}}(\bar{x}, \cdot)$ and hence a.s. $\bar{X}_{t} \in J H$.

Theorem 4.1 The process $\left\{\bar{X}_{t}: t \geq 0\right\}$ defined by (4.6) is a càdlàg strong Markov process with transition semigroup $\left(Q_{t}^{\bar{\mu}}\right)_{t \geq 0}$.

Proof. By the construction (4.6), $\left\{\bar{X}_{t}: t \geq 0\right\}$ is adapted to the filtration $\mathcal{F}_{t}:=\sigma\left(\left\{\tilde{Y}_{s}: 0 \leq\right.\right.$ $s \leq t\})$. For $r, t \geq 0$,

$$
\begin{aligned}
\bar{X}_{r+t}-\bar{T}_{t} \bar{X}_{r} & =\tilde{Y}_{r+t}-\bar{T}_{t} \tilde{Y}_{r}+\int_{r}^{r+t} \bar{T}_{r+t-s} \bar{A} \tilde{Y}_{s} d s \\
& =\left(\tilde{Y}_{r+t}-\tilde{Y}_{r}\right)+\int_{r}^{r+t} \bar{T}_{r+t-s} \bar{A}\left(\tilde{Y}_{s}-\tilde{Y}_{r}\right) d s
\end{aligned}
$$

Since $\left\{\tilde{Y}_{r+t}-\tilde{Y}_{r}: t \geq 0\right\}$ given $\mathcal{F}_{r}$ is a process with independent increments and has the same law as $\left\{\tilde{Y}_{t}: t \geq 0\right\}$, Lemma 4.3 implies that

$$
\boldsymbol{E}\left[\exp \left\{i\left\langle\bar{X}_{r+t}, \bar{a}\right\rangle_{-}\right\} \mid \mathcal{F}_{r}\right]=\exp \left\{i\left\langle\bar{X}_{r}, \bar{T}_{t}^{*} \bar{a}\right\rangle_{-}-\int_{0}^{t} \tilde{\psi}_{0}\left(\bar{T}_{s}^{*} \bar{a}\right) d s\right\} .
$$

Thus $\left\{\bar{X}_{t}: t \geq 0\right\}$ is a Markov process with transition semigroup $\left(Q_{t}^{\bar{\mu}}\right)_{t \geq 0}$. The strong Markov property holds since $\left(Q_{t}^{\bar{\mu}}\right)_{t \geq 0}$ is Feller.

Now let $\bar{x}=J x$ for some $x \in H$. In this case, $\bar{X}_{t} \in J H$ a.s. by Lemma 4.3. Recall that $J: H \rightarrow J H \subset \tilde{H} \subset \bar{H}$ and let $X_{t}=1_{J H}\left(\bar{X}_{t}\right) J^{-1}\left(\bar{X}_{t}\right)$, where $J^{-1}: J H \rightarrow H$ denotes the inverse map of $J$. Then $\left\{X_{t}: t \geq 0\right\}$ is an OU-process with transition semigroup $\left(Q_{t}^{\mu}\right)_{t \geq 0}$ and $X_{0}=x$. This gives a construction of the OU-process $\left\{X_{t}: t \geq 0\right\}$ from the càdlàg strong Markov process $\left\{\bar{X}_{t}: t \geq 0\right\}$. In general, $\left\{X_{t}: t \geq 0\right\}$ does not have right continuous modification in $H$. A similar construction in the measure-valued setting has been used in [6] to prove the non-existence of right continuous realization of a general immigration process. 


\section{Brownian transition semigroups}

We have seen that a general SC-semigroup on $H$ can always be extended to a differentiable one in the entrance space $\tilde{H}$ and a càdlàg realization of the corresponding OU-process can always be constructed in an extension $\bar{H}$ of $\tilde{H}$. In this section, we provided some explicit characterization for the non-negative elements of $\tilde{L}^{2}\left(\mathbb{R}^{d}\right)$ and $\tilde{L}^{2}(0, \infty)$ constructed respectively from $L^{2}\left(\mathbb{R}^{d}\right)$ and $L^{2}(0, \infty)$. It seems that the explicit characterization for all elements of $\bar{L}^{2}\left(\mathbb{R}^{d}\right)$ and $\bar{L}^{2}(0, \infty)$ is much more sophisticated.

We first consider the case where $\left(T_{t}\right)_{t \geq 0}$ is the transition semigroup of the standard Brownian motion on $\mathbb{R}^{d}$. Let $\tilde{H}:=\tilde{L}^{2}\left(\mathbb{R}^{d}\right)$ be defined from $H:=L^{2}\left(\mathbb{R}^{d}\right)$ and $\left(T_{t}\right)_{t \geq 0}$. Let

$$
g_{d}(s, x)=\frac{1}{(2 \pi s)^{d / 2}} \exp \left\{-|x|^{2} / 2 s\right\}, \quad s>0, x \in \mathbb{R}^{d},
$$

where $|\cdot|$ denote the Euclidean norm on $\mathbb{R}^{d}$, and let $S\left(\mathbb{R}^{d}\right)$ be the set of signed-measures $\mu$ on $\mathbb{R}^{d}$ with total variation measures $\|\mu\|$ satisfying

$$
\int_{0}^{l} d s \int_{\mathbb{R}^{d}}\|\mu\|(d x) \int_{\mathbb{R}^{d}} g_{d}(2 s, y-x)\|\mu\|(d y)<\infty
$$

for some $l>0$. Let $S_{+}\left(\mathbb{R}^{d}\right)$ and $\tilde{L}_{+}^{2}\left(\mathbb{R}^{d}\right)$ denote respectively the subsets of non-negative elements of $S\left(\mathbb{R}^{d}\right)$ and $\tilde{L}^{2}\left(\mathbb{R}^{d}\right)$.

Theorem 5.1 There is a 1-1 correspondence between $\tilde{x} \in \tilde{L}_{+}^{2}\left(\mathbb{R}^{d}\right)$ and $\mu \in S_{+}\left(\mathbb{R}^{d}\right)$ which is given by

$$
\tilde{x}(s, \cdot)=\int_{\mathbb{R}^{d}} g_{d}(s, \cdot-z) \mu(d z), \quad s>0 .
$$

Proof. If $\mu \in S_{+}\left(\mathbb{R}^{d}\right)$, then (5.3) defines a non-negative entrance path $\tilde{x}$ for $\left(T_{t}\right)_{t \geq 0}$. Since

$$
\begin{aligned}
\int_{0}^{l}\|\tilde{x}(s, \cdot)\|^{2} d s & =\int_{0}^{l} d s \int_{\mathbb{R}^{d}}\left(\int_{\mathbb{R}^{d}} g_{d}(s, y-z) \mu(d z)\right)^{2} d y \\
& =\int_{0}^{l} d s \int_{\mathbb{R}^{d}} d y \int_{\mathbb{R}^{d}} g_{d}(s, y-x) \mu(d x) \int_{\mathbb{R}^{d}} g_{s}(y-z) \mu(d z) \\
& =\int_{0}^{l} d s \int_{\mathbb{R}^{d}} \mu(d x) \int_{\mathbb{R}^{d}} g_{d}(2 s, z-x) \mu(d z) \\
& <\infty
\end{aligned}
$$

we have $\tilde{x} \in \tilde{L}_{+}^{2}\left(\mathbb{R}^{d}\right)$. Conversely, suppose that $\tilde{x} \in \tilde{L}_{+}^{2}\left(\mathbb{R}^{d}\right)$ and let $\kappa_{s}(d y)=\tilde{x}(s, y) d y$. Then $\left(\kappa_{s}\right)_{s>0}$ is a measure-valued entrance law for $\left(T_{t}\right)_{t \geq 0}$. By the property of the Brownian semigroup, there is a measure $\mu$ on $\mathbb{R}^{d}$ such that $\kappa_{s}=\mu T_{s}$; see e.g. Dynkin [3, p.80]. Thus $\tilde{x}(s, \cdot)$ has the representation (5.3), and (5.2) follows from (2.2) and the calculations above.

When $d=1$, we can give a necessary and sufficient condition for (5.2). Observe that for $0<l \leq 1$ we have

$$
\int_{0}^{l} g_{1}(2 s, y-x) d s<\int_{0}^{1} \frac{1}{2 \sqrt{\pi s}} \exp \left\{-(y-x)^{2} / 4\right\} d s=\frac{1}{\sqrt{\pi}} \exp \left\{-(y-x)^{2} / 4\right\}
$$


and for $l>1$ we have

$$
\int_{0}^{l} g_{1}(2 s, y-x) d s>\int_{1}^{l} \frac{1}{2 \sqrt{\pi l}} \exp \left\{-(y-x)^{2} / 4\right\} d s=\frac{l-1}{2 \sqrt{\pi l}} \exp \left\{-(y-x)^{2} / 4\right\} .
$$

By Lemma 2.1 and the proof of Theorem 5.1, (5.2) holds if and only if

$$
\int_{\mathbb{R}}\|\mu\|(d x) \int_{\mathbb{R}} \exp \left\{-(y-x)^{2} / 4\right\}\|\mu\|(d y)<\infty .
$$

Theorem 5.1 gives a complete characterization of non-negative elements of $\tilde{L}^{2}\left(\mathbb{R}^{d}\right)$. By this result, (5.3) also defines an element of $\tilde{L}^{2}\left(\mathbb{R}^{d}\right)$ for $\mu \in S\left(\mathbb{R}^{d}\right)$. Unfortunately, this representation does not give all elements of $\tilde{L}^{2}\left(\mathbb{R}^{d}\right)$. To see this, take any sequence $\left\{a_{k}\right\} \subset \mathbb{R}$ and observe that

$$
\int_{0}^{\infty} e^{-2 b s} d s \int_{\mathbb{R}}\left[g_{1}(s, y-x)-g_{1}(s, y)\right]^{2} d y \rightarrow 0
$$

as $x \rightarrow 0$. Then for each $k \geq 1$ there exists $\varepsilon_{k} \in\left(0, k^{-2}\right)$ such that

$$
a_{k}^{2} \int_{0}^{\infty} e^{-2 b s} d s \int_{\mathbb{R}}\left[g_{1}\left(s, y-\varepsilon_{k}\right)-g_{1}(s, y)\right]^{2} d y \leq 2^{-k} .
$$

Let $x_{k}=k^{-1}$ and $z_{k}=k^{-1}+\varepsilon_{k}$. Then $z_{k}>x_{k}>z_{k+1}>x_{k+1}>\ldots$ decrease to zero. By (5.5) and the shift invariance of the Lebesgue measure it is easy to see that

$$
\tilde{x}_{n}(s, \cdot)=\sum_{k=1}^{n} a_{k}\left[g_{1}\left(s, \cdot-z_{k}\right)-g_{1}\left(s, \cdot-x_{k}\right)\right], \quad s>0,
$$

defines a Cauchy sequence $\left\{\tilde{x}_{n}\right\} \subset \tilde{L}^{2}(\mathbb{R})$ with limit $\tilde{x} \in \tilde{L}^{2}(\mathbb{R})$ given by

$$
\tilde{x}(s, \cdot)=\sum_{k=1}^{\infty} a_{k}\left[g_{1}\left(s, \cdot-z_{k}\right)-g_{1}\left(s, \cdot-x_{k}\right)\right], \quad s>0 .
$$

To represent this element in the form of (5.3) we need to let

$$
\mu=\sum_{k=1}^{\infty} a_{k} \delta_{z_{k}}-\sum_{k=1}^{\infty} a_{k} \delta_{x_{k}}
$$

which is clearly not belonging to $S(\mathbb{R})$ in general.

Now we consider the case where $\left(T_{t}\right)_{t \geq 0}$ is the transition semigroup of the absorbing barrier Brownian motion in $D=(0, \infty)$. Let $\gamma(d y)=\left(1-e^{-y^{2}}\right) d y$ and let $\tilde{L}^{2}(D, \gamma)$ be defined from $L^{2}(D, \gamma)$ and $\left(T_{t}\right)_{t \geq 0}$. Let

$$
p_{s}(x, y)=g_{1}(s, y-x)-g_{1}(s, y+x), \quad s, x, y>0,
$$

and let

$$
k_{s}(y)=\left.2^{-1}(d / d x) p_{s}(x, y)\right|_{x=0^{+}}=y g_{1}(s, y) / s, \quad s, y>0 .
$$

Let $S(D, \gamma)$ be the set of signed-measures $\mu$ on $D$ with total variation measures $|\mu|$ satisfying

$$
\int_{0}^{l} d s \int_{D}\left(\int_{D} p_{s}(x, y)|\mu|(d x)\right)^{2} \gamma(d y)<\infty
$$

for some $l>0$. Let $S_{+}(D, \gamma)$ and $\tilde{L}_{+}^{2}(D, \gamma)$ denote respectively the subsets of non-negative elements of $S(D, \gamma)$ and $\tilde{L}^{2}(D, \gamma)$. 
Theorem 5.2 There is a 1-1 correspondence between $\tilde{x} \in \tilde{L}_{+}^{2}(D, \gamma)$ and $(a, \mu) \in[0, \infty) \times$ $S_{+}(D, \gamma)$ which is given by

$$
\tilde{x}(s, \cdot)=a k_{s}(\cdot)+\int_{D} p_{s}(z, \cdot) \mu(d z), \quad s>0 .
$$

Proof. If $(a, \mu) \in[0, \infty) \times S_{+}(D, \gamma)$, then (5.10) defines an entrance path $\tilde{x}$ for $\left(T_{t}\right)_{t \geq 0}$. Since

$$
\int_{0}^{l} k_{s}(y)^{2} d s \leq \int_{0}^{\infty} \frac{y^{2}}{2 \pi s^{3}} e^{-y^{2} / s} d s=\frac{1}{2 \pi y^{2}}
$$

we have

$$
\begin{aligned}
\int_{0}^{l}\|\tilde{x}(s, \cdot)\|^{2} d s= & \int_{0}^{l} d s \int_{D}\left(a k_{s}(y)+\int_{D} p_{s}(z, y) \mu(d z)\right)^{2} \gamma(d y) \\
\leq & 2 a^{2} \int_{0}^{l} d s \int_{D} k_{s}(y)^{2} \gamma(d y) \\
& +2 \int_{0}^{l} d s \int_{D}\left(\int_{D} p_{s}(z, y) \mu(d z)\right)^{2} \gamma(d y) \\
< & \infty
\end{aligned}
$$

that is, $\tilde{x} \in \tilde{L}_{+}^{2}(D, \gamma)$. Conversely, suppose that $\tilde{x} \in \tilde{L}_{+}^{2}(\mathbb{R})$ and let $\kappa_{s}(d y)=\tilde{x}(s, y) d y$. Then $\left(\kappa_{s}\right)_{s>0}$ is a measure-valued entrance law for $\left(T_{t}\right)_{t \geq 0}$. By the property of the absorbing barrier Brownian motion, there is a constant $a \geq 0$ and a measure $\mu$ on $D$ such that (5.10) holds; see e.g. [9, Lemma 1.1]. Since

$$
\int_{0}^{l} d s \int_{D}\left(\int_{D} p_{s}(z, y) \mu(d z)\right)^{2} \gamma(d y) \leq \int_{0}^{l}\|\tilde{x}(s, \cdot)\|^{2} d s,<\infty .
$$

we have $\mu \in S(D, \gamma)$.

By the general results of section 4, an OU-process associated with the absorbing barrier Brownian motion in $D=(0, \infty)$ always has càdlàg realization in $\bar{L}^{2}(D, \gamma) \supset \tilde{L}^{2}(D, \gamma)$ defined from $L^{2}(D, \gamma)$. It was observed in [?] that in a special case the process also has càdlàg realization in $S(D, \gamma)$.

Acknowledgement We thank Klaus Fleischmann and a referee for their careful reading of the manuscript and helpful comments.

\section{References}

[1] Bogachev, V.I., Röckner, M. and Schmuland, B., Generalized Mehler semigroups and applications, Probab. Th. Rel. Fields 105 (1996), 193-225.

[2] Dawson, D.A., Li, Z.H., Schmuland, B. and Sun, W., Generalized Mehler semigroups and catalytic branching processes with immigration, Potential Anal. 21, 75-97.

[3] Dynkin, E.B.: Three classes of infinite dimensional diffusion processes, J. Funct. Anal. 86 (1989), 75-110. 
[4] Fuhrman, M. and Röckner, M., Generalized Mehler semigroups: The non-Gaussian case, Potential Anal. 12 (2000), 1-47.

[5] Li, Z.H., Convolution semigroups associated with measure-valued branching processes, Chinese Science Bulletin (Chinese Edition) 40 (1995), 2018-2021 / (English Edition) 41 (1996), 276-280.

[6] Li, Z.H., Immigration structures associated with Dawson-Watanabe superprocesses, Stochastic Process. Appl. 62 (1996), 73-86.

[7] Li, Z.H., Immigration processes associated with branching particle systems, Adv. Appl. Probab. 30 (1998), 657-675.

[8] Li, Z.H., Skew convolution semigroups and related immigration processes, Theory Probab. Appl. 46 (2002), 274-296.

[9] Li, Z.H. and Shiga T., Measure-valued branching diffusions: immigrations, excursions and limit theorems, J. Math. Kyoto Univ. 35 (1995), 233-274.

[10] Linde, W., Probability in Banach Spaces - Stable and Infinitely Divisible Distributions, Wiley, New York (1986).

[11] van Neerven, J. M. A. M., Continuity and representation of Gaussian Mehler semigroups, Potential Anal. 13 (2000), 199-211.

[12] Schmuland, B. and Sun W., On the equation $\mu_{t+s}=\mu_{s} * T_{s} \mu_{t}$, Statist. Probab. Letters, 52 (2001), 183-188.

[13] Sharpe, M.J., General Theory of Markov Processes, Academic Press, New York (1988). 